\title{
Genome-wide miRNA profiling of villus and decidua of recurrent spontaneous abortion patients
}

\author{
Fulu Dong ${ }^{*}$, Yuan Zhang ${ }^{1, *}$, Fei Xia, Yi Yang, Sidong Xiong, Liping Jin ${ }^{1}$ and Jinping Zhang \\ Institutes of Biology and Medical Sciences, Soochow University, Suzhou, Jiangsu Province 215123, People's Republic \\ of China and ${ }^{1}$ Laboratory for Reproductive Immunology, Hospital and Institute of Obstetrics and Gynecology, \\ Fudan University Shanghai Medical College, No. 413, Zhaozhou Road, Shanghai 200011, People's Republic of China
} Correspondence should be addressed to J Zhang; Email: j_pzhang@suda.edu.cn or to L Jin; jinliping2012@gmail.com

*(F Dong and Y Zhang contributed equally to this work)

\begin{abstract}
MicroRNAs (miRNAs) are non-coding RNA molecules of about 22 nucleotides that involved in post-transcriptional gene regulation. Evidence indicates that miRNAs play essential roles in endometriosis, pre-eclampsia, infertility and other reproductive system diseases. However, whether miRNAs are involved in recurrent spontaneous abortion (RSA) is unclear. In this work, we analysed the miRNA expression profiles in six pairs of villus or decidua from RSA patients and normal pregnancy (NP) women using a human miRNA microarray. Some of the chip results were confirmed by RT-qPCR. In the villi of RSA patients, expression of hsa-miR-184, hsa-miR-187 and hsa-miR-125b-2 was significantly higher, while expression of hsa-miR-520f, hsa-miR-3175 and hsa-miR-4672 was significantly lower, comparing with those of NP control. As well, a total of five miRNAs (hsa-miR-517c, hsa-miR-519a-1, hsa-miR-522, hsa-miR-520h and hsa-miR-184) were upregulated in the decidua of RSA patients. The target genes of these differentially expressed miRNAs were predicted by miRWalk, and we speculate a network of miRNA regulating RSA by target genes function on adhesion, apoptosis and angiogenesis. Our study may help clarify the molecular mechanisms which are involved in the progression of RSA, and provide a reference for future research.

Reproduction (2014) 148 33-41
\end{abstract}

\section{Introduction}

Recurrent spontaneous abortion (RSA) has been defined as the miscarriage of two or more consecutive pregnancies before 20 weeks of pregnancy in human (Rai \& Regan 2006), which is difficult to treat in the clinical setting. About $1 \%$ of couples are affected by recurrent miscarriage. In the female, the causes of RSA have been generally recognised including genetics, anatomical, endocrine, placental anomalies, hormonal problems, infection, smoking and alcohol consumption, exposure to environmental factors, psychological trauma and stressful life event, certain coagulation and immunoregulatory protein defects (Pandey et al. 2005). However, half of the patients with RSA still cannot find the cause of recurrent miscarriage (Pandey et al. 2005).

MicroRNAs (miRNAs) is a class of endogenous gene encoding a length of about 22 nucleotides with noncoding single-stranded RNA molecules. In 1993, Lee et al. (1993) and Wightman et al. (1993) first discovered miRNA in a worm, called lin-4. So far, miRNA-regulating gene expression has been observed in almost all multicellular eukaryotes and many of the unicellular eukaryotes. Also, it has been demonstrated that miRNA has a critical regulatory role in endometriosis (Pan et al. 2007), pre-eclampsia (Zhang et al. 2010), infertility (McCallie et al. 2010) and other reproductive system diseases. miR-133a can reduce RSA, and is regulated by human leukocyte antigen HLA-G gene (Wang et al. 2012). Some miRNA polymorphisms (miR-146aC $>$ G, miR-149T $>$ C, miR-196a2T $>C$ and miR-499A $>$ G) do have a risk of RSA in Korean women (Jeon et al. $2012 a, b)$. However, the genome-wide miRNA profiles in RSA patients and the functional mechanisms of these differentially expressed miRNAs are largely unclear.

Therefore, the present study was undertaken to investigate the expression pattern and possible target genes of miRNA in the villi and decidua from women who suffered from RSA.

\section{Materials and methods}

\section{Patients and samples}

All subjects in this study are Chinese. The decidual tissues and villus tissues were obtained from 15 clinically normal 
Table 1 Primers used in real-time PCRs for detecting microRNAs expression.

\begin{tabular}{|c|c|}
\hline Gene name & Primer sequence $\left(5^{\prime}-3^{\prime}\right)$ \\
\hline \multirow{3}{*}{ miR-184 } & F: ACACTCCAGCTGGGTGGACGGAGAACTGAT \\
\hline & R: CTCAACTGGTGTCGTGGA \\
\hline & RT primer: CTCAACTGGTGTCGTGGAGTCGGCAATTCAGTTGAGACCCTTAT \\
\hline \multirow[t]{3}{*}{ miR-572 } & F: ACACTCCAGCTGGGGTCCGCTCGGCGGTGG \\
\hline & R: CTCAACTGGTGTCGTGGA \\
\hline & RT primer: CTCAACTGGTGTCGTGGAGTCGGCAATTCAGTTGAGGGCCACCG \\
\hline \multirow[t]{3}{*}{ miR-187 } & F: ACACTCCAGCTGGGTCGTGTCTTGTGTTGC \\
\hline & R: CTCAACTGGTGTCGTGGA \\
\hline & RT primer: CTCAACTGGTGTCGTGGAGTCGGCAATTCAGTTGAGCCGGCTGC \\
\hline \multirow[t]{3}{*}{ miR-125b } & F: ACACTCCAGCTGGGTCACAAGTCAGGCTCT \\
\hline & R: CTCAACTGGTGTCGTGGA \\
\hline & RT primer: CTCAACTGGTGTCGTGGAGTCGGCAATTCAGTTGAGGTCCCAAG \\
\hline \multirow[t]{3}{*}{ miR-517c } & F: ACACTCCAGCTGGGATCGTGCATCCTTTTA \\
\hline & R: CTCAACTGGTGTCGTGGA \\
\hline & RT primer: CTCAACTGGTGTCGTGGAGTCGGCAATTCAGTTGAGACACTCTA \\
\hline \multirow[t]{3}{*}{ miR-519a } & F: ACACTCCAGCTGGGAAAGTGCATCCTTTTA \\
\hline & R: CTCAACTGGTGTCGTGGA \\
\hline & RT primer: СТСААСTGGTGTCGTGGAGTCGGCAАTTCAGTTGAGACAСTCTA \\
\hline \multirow[t]{3}{*}{ miR-522 } & F: ACACTCCAGCTGGGAAAATGGTTCCСTTTA \\
\hline & R: CTCAACTGGTGTCGTGGA \\
\hline & RT primer: CTCAACTGGTGTCGTGGAGTCGGCAATTCAGTTGAGACACTCTA \\
\hline \multirow[t]{3}{*}{ miR-520h } & F: ACACTCCAGCTGGGACAAAGTGCTTCCCTT \\
\hline & R: CTCAACTGGTGTCGTGGA \\
\hline & RT primer: CTCAACTGGTGTCGTGGAGTCGGCAATTCAGTTGAGACTCTAAA \\
\hline \multirow[t]{3}{*}{ miR-1247 } & F: ACACTCCAGCTGGGACCCGTCCCGTTCGTC \\
\hline & R: CTCAACTGGTGTCGTGGA \\
\hline & RT primer: CTCAACTGGTGTCGTGGAGTCGGCAATTCAGTTGAGTCCGGGGA \\
\hline \multirow[t]{3}{*}{ miR-1238 } & F: ACACTCCAGCTGGGCTTCCTCGTCTGTCTG \\
\hline & R: CTCAACTGGTGTCGTGGA \\
\hline & RT primer: CTCAACTGGTGTCGTGGAGTCGGCAATTCAGTTGAGGGGGCAGA \\
\hline \multirow[t]{2}{*}{$\beta$-actin } & F: GGCACCACCATGTACCCTGGCAT \\
\hline & R: TCCTGCTTGCTGATCCACATCTGCT \\
\hline
\end{tabular}

pregnancies (NP) that were detected by ultrasound (age: $27.30 \pm 1.31$ years and gestational age at sampling 7.26 \pm 1.43 weeks (mean \pm s.D.)), which were terminated for non-medical reasons, and 20 RSA cases (age: 28.37 \pm 1.46 years and gestational age at sampling $7.53 \pm 1.52$ weeks (mean \pm s.D.)), which were classified as having unexplained aetiology for the occurrence of abortion after the exclusion of maternal anatomic or hormonal abnormality, paternal and maternal chromosomal abnormality, and infection. All the tissues were minced into small fragments and incubated in TRIzol reagent and stored at $-80{ }^{\circ} \mathrm{C}$. Each patient completed a signed, written consent form, and the form has been approved by Human Investigation Committee of Hospital of Obstetrics \& Gynecology, Fudan University.

\section{Microarray analysis}

Total RNAs were extracted using TRIzol reagent (Invitrogen) according to the manufacturer's description, quantified using the NanoDrop ND-1000 and the RNA integrity was assessed using standard denaturing agarose gel electrophoresis, purified using the miRVana miRNA Isolation Kit (Ambion, Austin, TX, USA), tailed with polyadenylation polymerase, ligated with biotinylated DNA dendrimers, and hybridised to Affymetrix GeneChip miRNA arrays using the FlashTag Biotin RNA Labeling Kit (Genisphere, Hatfield, PA, USA) according to the manufacturer's instructions. Slides were scanned with the Affymetrix GeneChip Scanner 3000 (Affymetrix, Santa Clara, CA, USA), and miRNA data were analysed using the miRNA QC Tool (Affymetrix).
A
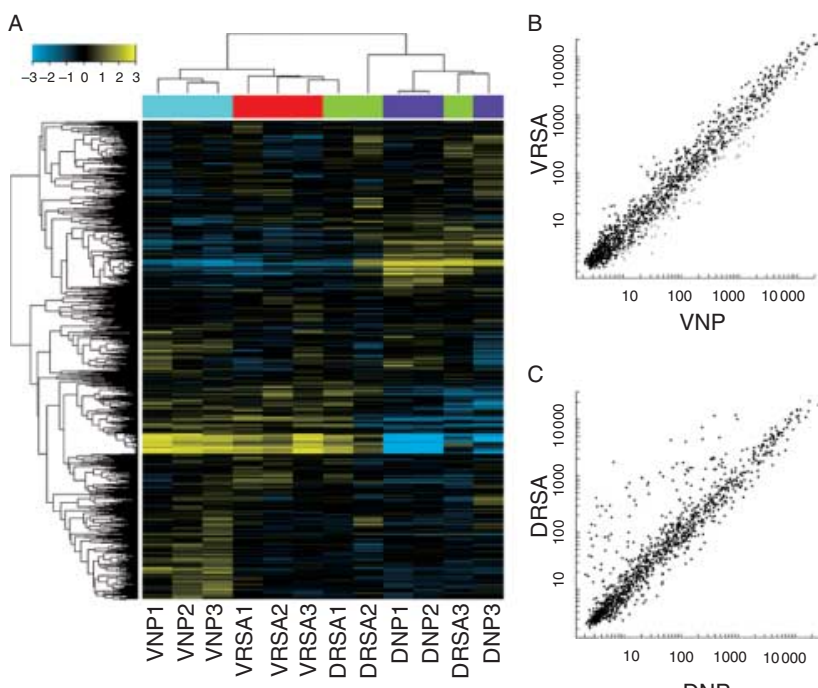

C

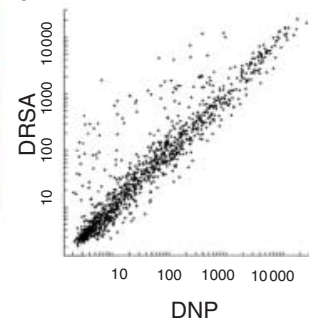

Figure 1 The expression profiles of global miRNAs in the villus and decidua of RSA patients or controls. (A) Heatmap of global miRNAs in villus or decidua from 12 people (three VRSA and three DRSA from six RSA patients, three VNP and three DNP from six NP controls).

(B) Log-log scatter plot comparing global miRNAs expression profiles in villus of RSA patients with those of NP controls. (C) Log-log scatter plot comparing global miRNAs expression profiles in decidua of RSA patients with those of NP controls. VNP, villus of NP; DNP, decidua of NP; VRSA, villus of RSA; DRSA, decidua of RSA. 
Table 2 Differentially expressed miRNAs in villi and decidua from RSA patients vs controls.

\begin{tabular}{|c|c|c|c|c|}
\hline miRNA & $\begin{array}{c}\text { Fold- } \\
\text { changed }\end{array}$ & Style & $q$ value & Score $(d)$ \\
\hline \multicolumn{5}{|c|}{ Six differentially expressed miRNAs in villi } \\
\hline hsa-miR-184_st & 5.5926 & Up & 1.5933525 & 3.8200033 \\
\hline hsa-miR-187_st & 5.3167 & Up & 0 & 4.5640246 \\
\hline $\begin{array}{l}\text { hsa-miR-125b- } \\
\text { 2-star_st }\end{array}$ & 5.0732 & Up & 1.5933525 & 4.2958393 \\
\hline hsa-miR-520f_st & 0.1568 & Down & 2.183483 & -2.701141 \\
\hline hsa-miR-3175_st & 0.1514 & Down & 0 & -4.026834 \\
\hline hsa-miR-4672_st & 0.1212 & Down & 0 & -5.410953 \\
\hline \multicolumn{5}{|c|}{ Five differentially expressed miRNAs in decidua } \\
\hline hsa-miR-517c_st & 92.0394 & Up & 0 & 7.2452875 \\
\hline hsa-miR-519a_st & 42.3243 & Up & 0 & 5.8489197 \\
\hline hsa-miR-522_st & 38.3829 & Up & 0 & 9.0965277 \\
\hline hsa-miR-520h_st & 33.095 & Up & 0 & 6.6350494 \\
\hline hsa-miR-184_st & 15.8567 & Up & 0 & 6.1824566 \\
\hline
\end{tabular}

$q$ value $\leq 5$

\section{Real-time PCR}

Total RNAs were purified using TRIzol reagent (Invitrogen) and reverse transcribed using a RT system (Promega) according to the manufacturer's description. Real-time PCR was carried out using FastStart Universal SYBR Green Master (Roche
Diagnostics) and analysed using eppendorf Real-Time Detection System (Eppendorf, Hauppauge, NY, USA). The primer pairs used for miRNAs and $\beta$-actin are shown in Table 1. PCR parameters were as follows: $95{ }^{\circ} \mathrm{C}$ for $5 \mathrm{~min}$, followed by 40 cycles of $95{ }^{\circ} \mathrm{C}$ for $15 \mathrm{~s}, 60{ }^{\circ} \mathrm{C}$ for $30 \mathrm{~s}$ and $72{ }^{\circ} \mathrm{C}$ for $30 \mathrm{~s}$. Relative expression level was calculated as $2^{-(\text {CT } \beta \text { act-CTgene) }}$ (Livak \& Schmittgen 2001). The miRNA level was normalised by the housekeeping gene, $\beta$-actin.

\section{Cluster analysis}

Unsupervised hierarchical clustering was carried out with average linkage and uncentred correlation as the similarity metrics using Cluster 3.0 (de Hoon et al. 2004). Heat maps were generated in Java Treeview. Data from each raw probe from the microarrays of all samples were averaged, and then the respective data from the samples was transformed as the provider divided by the average (mean). Relative expression of each miRNA was calculated via the ratio between the sample microarrays and the average of all microarrays (Sun et al. 2011). To draw a simple and perspicuity figure with the software, the relative expression of each gene was described as the $\log _{10}$ (ratio) in the heatmap figures from Cluster 3.0.
A

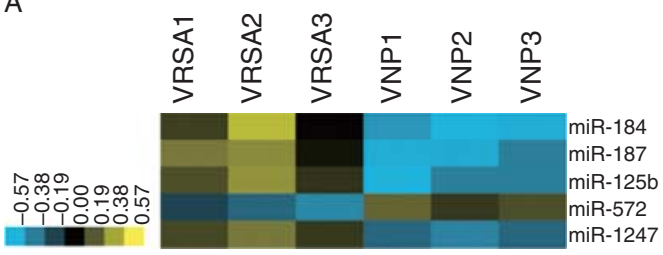

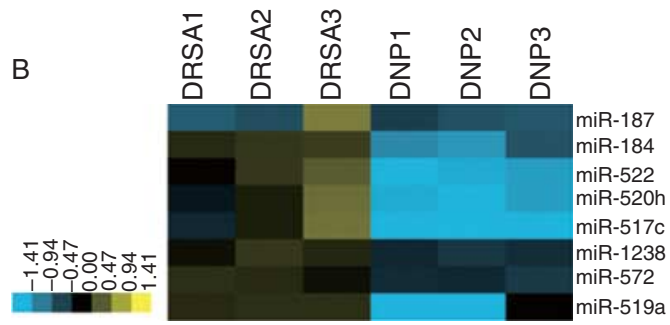

D
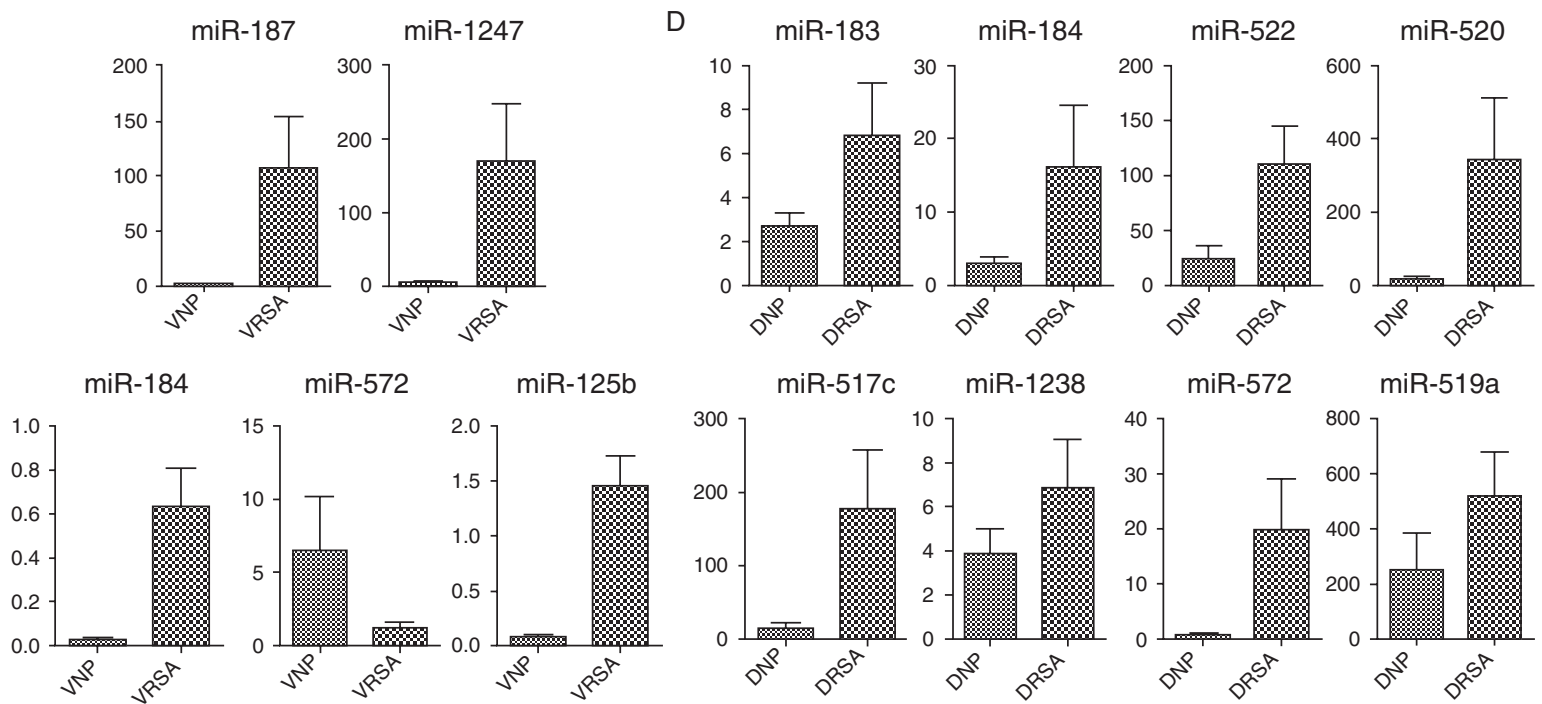

$\operatorname{miR}-125 b$

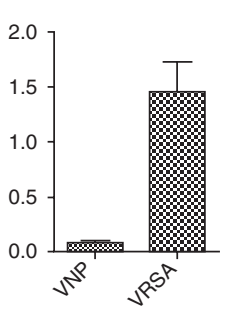

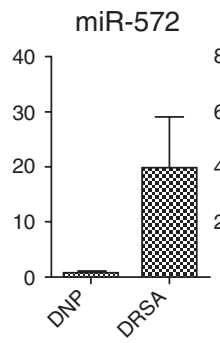

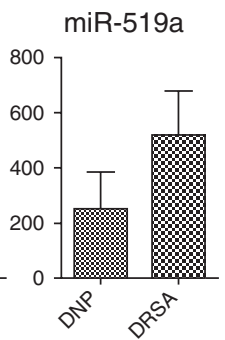

Figure 2 Expression change profile of ten miRNAs in villus or decidua of RSA patients and controls. (A and B) Heatmap of selected miRNAs in villus or decidua from 12 people (three VRSA and three DRSA from six RSA patients, three VNP and three DNP from six NP controls). (C and D) Expression pattern of selected miRNAs in villus or deciduas from 35 people (seven VRSA and 13 DRSA from 20 RSA patients, nine VNPand six DNP from 15 NP controls) detected by RT-qPCR. VNP, villus of NP; DNP, decidua of NP; VRSA, villus of RSA; DRSA, decidua of RSA. 


\section{Selection of differentially expressed miRNAs and functional analysis}

Differentially expressed miRNAs were identified using the significance analysis of microarrays (SAM) program. The miRNAs with $q$ value $\leq 0.05$, fold change $\geq 2$ or $\leq 0.5$ were considered to be significantly differentially expressed. Using miRWalk and Targetscan, we predicted the target genes of significant differentially expressed miRNAs, and then we selected genes which can act as target genes of two or more significant miRNAs to perform Gene Ontology (GO) and pathway analysis, the $-\log _{10}(P$ value) indicating the $\mathrm{GO}$ and pathway result was used in the histogram and line chart by SigmaPlot (Systat Software, Inc., San Jose, CA, USA).

\section{Results}

\section{Differential expression of miRNAs in villus and decidua of RSA patients}

In order to analyse the expression changes of miRNAs in the villus and decidua of RSA patients, we detected the global miRNA expression profiles of villus and decidua of RSA patients and NP controls (Fig. 1A and Supplementary Table 1 , see section on supplementary data given at the end of this article), using the R package from SAM to screen the differential expression of miRNAs following the criteria: $q$ value $\leq 0.05$; fold change $\geq 2$ or $\leq 0.5$. A total of 45 miRNAs were found to be differentially expressed, of which four miRNAs were upregulated, 41 miRNAs were downregulated in the villus of RSA patients compared with those in the villus of NP controls, and there are seven miRNAs upregulated in the decidua of RSA patients (Supplementary Table 2). We can intuitively observe this phenotype in log-log scatter plot (Fig. 1B and C). To further filter the key miRNA in RSA, we defined differential miRNAs as the criteria: $q$ value $\leq 0.05$; fold change $\geq 5$ or $\leq 0.2$. Three miRNAs (hsa-miR-184, hsa-miR-187 and hsa-miR-125b2) were found to exhibit higher specific expression and three miRNAs (hsa-miR-520f, hsa-miR-3175 and hsamiR-4672) lower specific expression in the villus of RSA patients compared with those in the villus of NP controls. Also, five miRNAs (hsa-miR-517c, hsa-miR-519a-1, hsamiR-522, hsa-miR-520h and hsa-miR-184) were upregulated in the decidua of RSA patients compared with those in NP controls. The detail information about some of these genes was showed in Table 2. We were surprised to find that hsa-miR-184 has a high expression level both in the villus and in decidua of RSA patients. This suggests that hsa-miR-184 may have a critical role in RSA. To examine the reliability of the array data, we selected ten miRNAs which were detected to have differential gene expression by microarray (Fig. 2A and B) to confirm their expression in villus or decidua of RSA and NP controls by using qPCR. The results of qPCRs were consistent with microarray data (Fig. 2C and D).

\section{Target prediction of differentially expressed miRNAs}

The target genes of these differentially expressed miRNAs were predicted by miRWalk (http://www.umm.uniheidelberg.de/apps/zmf/mirwalk/), which can integrate the predictive information of ten miRNA target prediction databases. If a gene can be predicted as a target gene by more than four databases simultaneously, we suppose that this target gene is effective. Next, we used these 'effective target genes' and differentially expressed miRNAs to establish the miRNA-gene Venn diagram (Fig. 3). Hsa-miR-184 has 431 target genes and hsa-miR-187 has 334 target genes predicted by miRWalk, and 20 of the target genes can be predicted as target genes of hsa-miR-184 and hsa-miR-187 (Fig. 3A). Hsa-miR-520f, hsa-miR-3175 and hsa-miR-4672 are downexpressed in the villus of RSA patients, and there are 179 target genes predicted by at least two of the three miRNAs (Fig. 3B). Hsa-miR-517c, hsa-miR-519a-1, hsa-miR-522, hsa-miR-520 and hsa-miR-184 are significantly overexpressed in the decidua of RSA patients, and there are 1547 target genes predicted by at least two of the five miRNAs (Fig. 3C).

\section{Functional prediction of differentially expressed miRNAs in RSA}

We selected genes which can be the target of two or more miRNAs simultaneously to perform GO and KEGG pathway analysis. The most significant biological
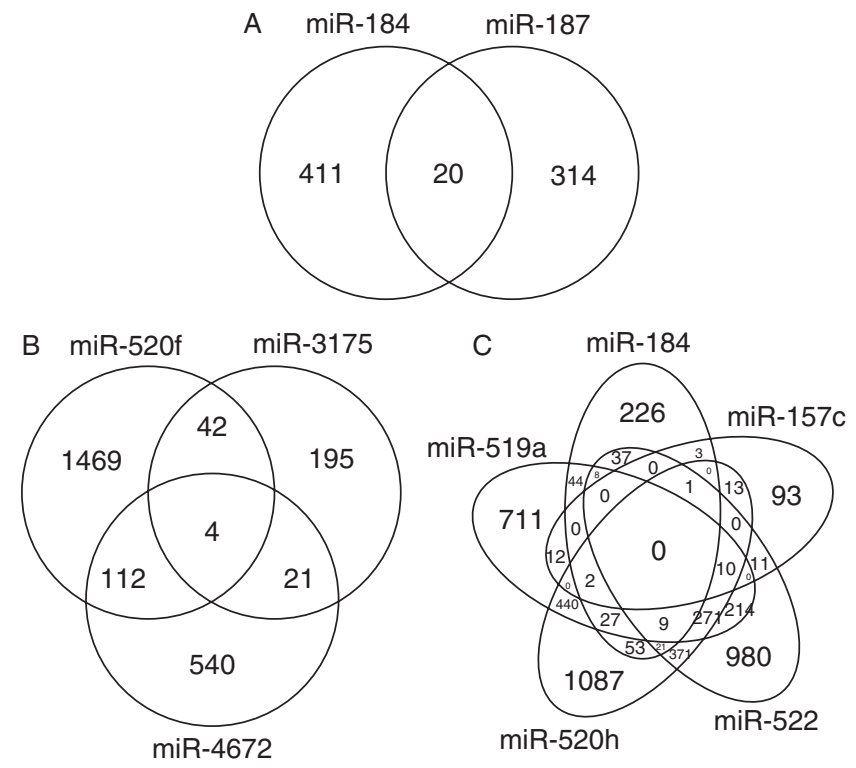

Figure 3 Venn diagram analysis of the relationship between the target genes predicted by differentially expressed miRNAs. (A) Relationship between the target genes predicted by hsa-mir-184 and hsa-mir-187. (B) Relationship between the target genes predicted by hsa-mir-520f, hsa-mir-3175 and hsa-mir-4672. (C) Relationship between the target genes predicted by hsa-mir-517c, hsa-mir-519a-1, hsa-mir-522, hsa-mir- 520h and hsa-mir-184. 
A

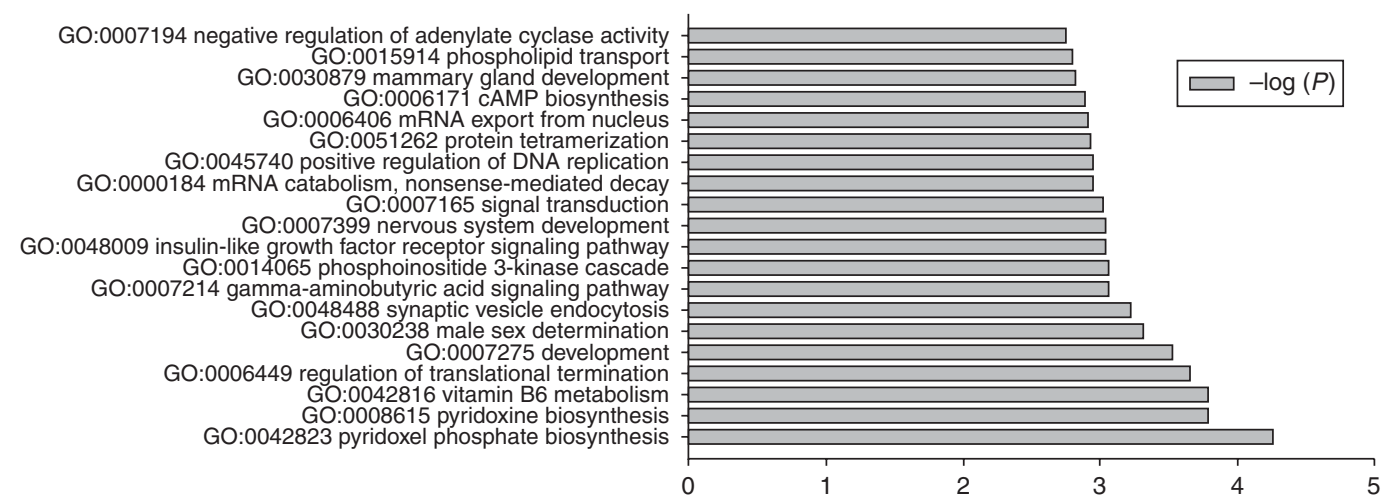

B

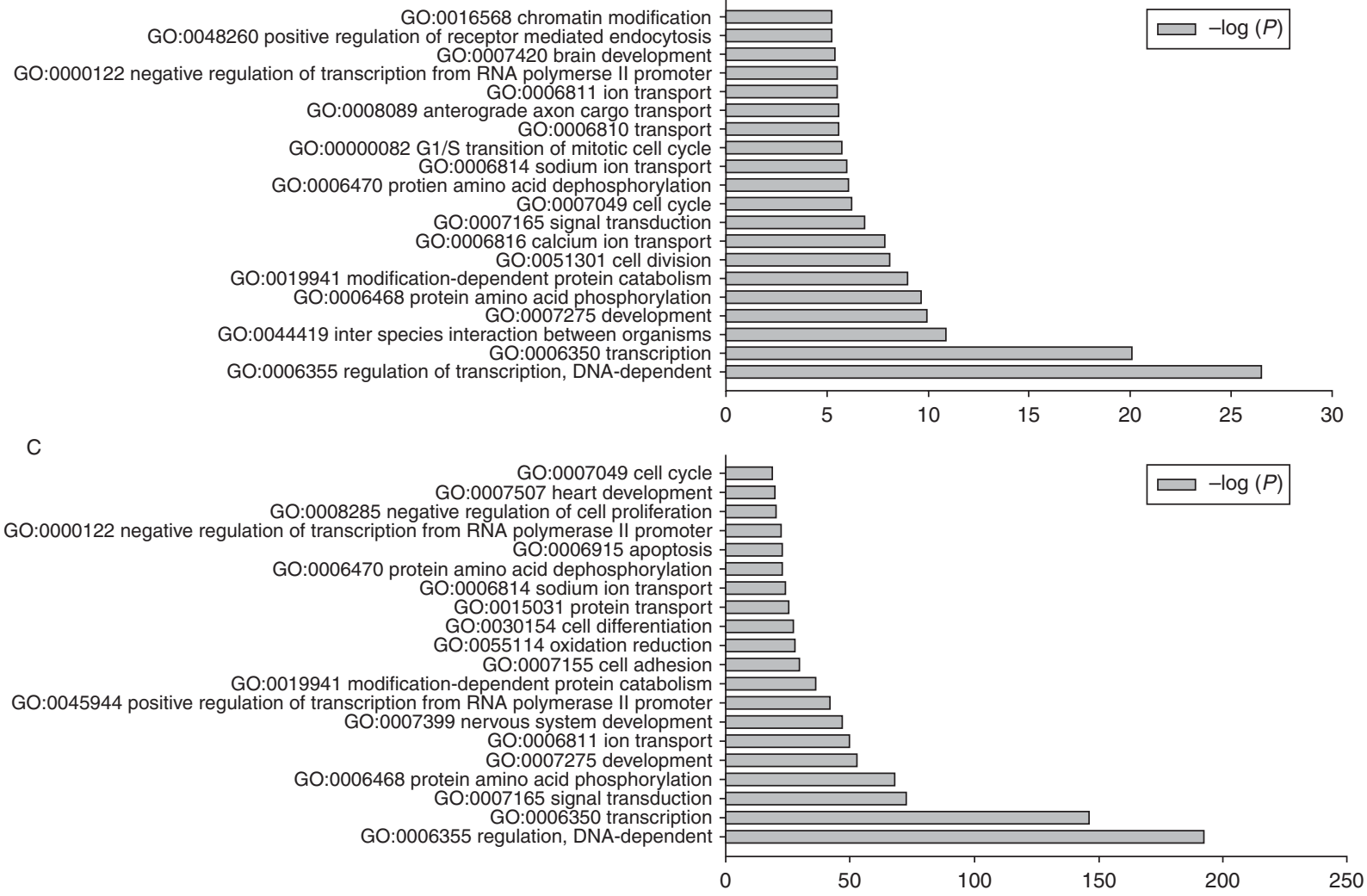

Figure 4 Result of the GO analysis of the target genes predicted by differentially expressed miRNAs. $-\log _{10}(P)$ indicates the GO score related to genes with the biological process by $P$ value. (A) Main biological processes of genes targeted by hsa-miR-184 and hsa-miR-187. (B) Main biological processes of genes targeted by two or more miRNAs of hsa-miR-520f, hsa-miR-3175 and hsa-miR-4672. (C) Main biological processes of genes targeted by two or more miRNAs of hsa-miR-517c, hsa-miR-519a-1, hsa-miR-522, hsa-miR-520h and hsa-miR-184.

processes of these target genes of overexpressed miRNAs in the villus of RSA patients were biosynthesis of pyridoxal phosphate and pyridoxine, metabolism of vitamin $\mathrm{B} 6$, regulation of translational termination, development, etc. (Fig. 4A). The most significant biological processes of the genes targeted by downexpressed miRNAs in the villus of RSA patients were regulation of transcription, DNA-dependent transcription, interspecies interaction between organisms, development, protein amino acid phosphorylation, etc.
(Fig. 4B). The most significant biological processes of these target genes of overexpressed miRNAs in the decidua of RSA patients were regulation of transcription, signal transduction, protein amino acid phosphorylation and development, etc. (Fig. 4C). Similarly, the pathway analysis showed that the predicted target genes in villus were involved in adherence junction, gap junction, purine metabolism, calcium signalling pathway, focal adhesion, endometrial cancer, etc, and the predicted target genes of high expressed miRNAs in the decidua of 
A

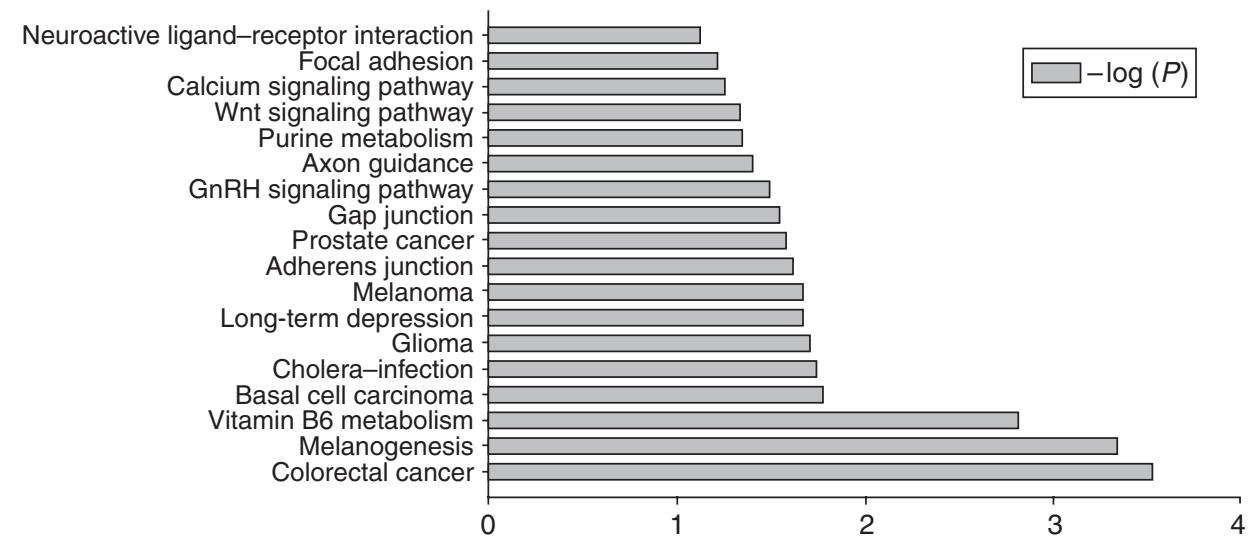

B

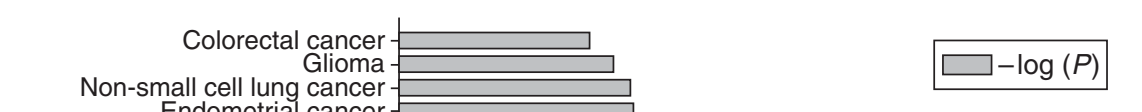

Endometria cancer

Tight junction Melanogenesis
Regulation of actin cytoskeleto Calcium signaling pathway Long-term potentiation Renal cell carcinoma Jak-STAT signaling pathway Wnt signaling pathway Ubiquitin mediated proteolysis mTOR signaling pathway Prostate cancer ErbB signaling pathway Small cell lung cancer Chronic myeloid leukemia Focal adhesion

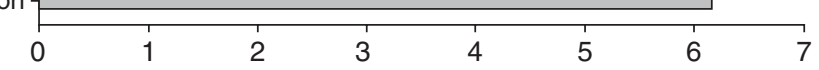

C

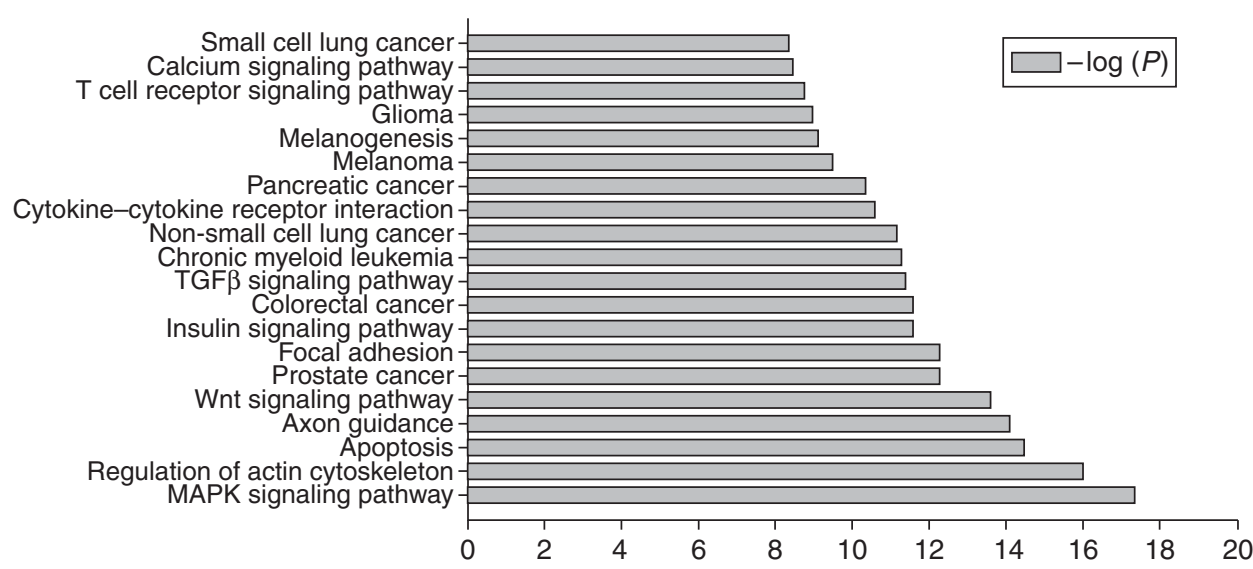

Figure 5 Result of pathway analysis of the target genes predicted by differentially expressed miRNAs. (A) Main pathway involved by genes targeted by hsa-mir-184 and hsa-mir-187. (B) Main pathway involved by genes targeted by two or more miRNAs of hsa-mir-520f, hsa-mir-3175 and hsa-mir-4672. (C) Main pathway involved by genes targeted by two or more miRNAs of hsa-mir-517c, hsa-mir-519a-1, hsa-mir-522, hsa-mir-520h and hsa-mir-184.

RSA patients were related to apoptosis, focal adhesion, T cell receptor signalling pathway, etc. (Fig. 5).

\section{Discussion}

Previous reports indicated that cell adhesion has a critical role in NP (Konijnenberg et al. 1997, Lyall et al. 1999, Chaiworapongsa et al. 2002), T cell adhesion to the extracellular matrix proteins, which was seen as a determinant of successful or failing pregnancy (Malgorzata \& Gorski 1998). In women with RSA, the adhesion of trophoblasts to endothelial cells is inhibited. However, the pathogenetic mechanism which inhibits the adhesion of trophoblast to endothelial cells remains unclear in women with RSA (Bulla et al. 1999). In the present study, we carried out GO and pathway analysis 
A

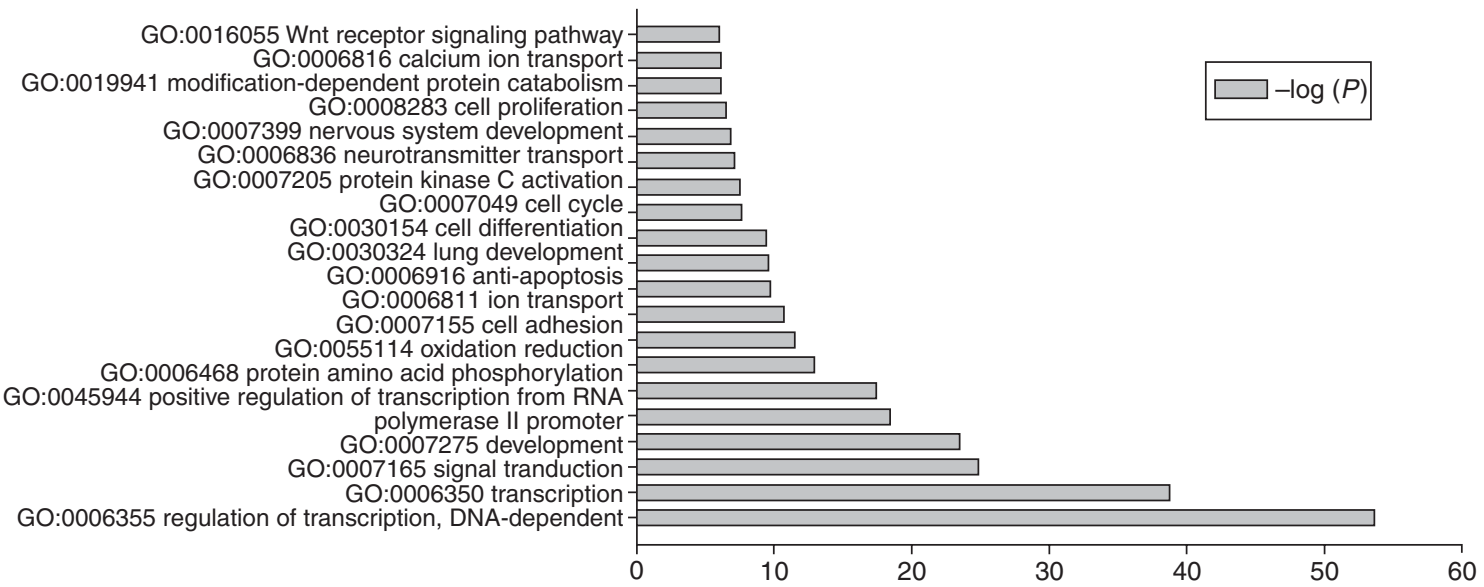

B

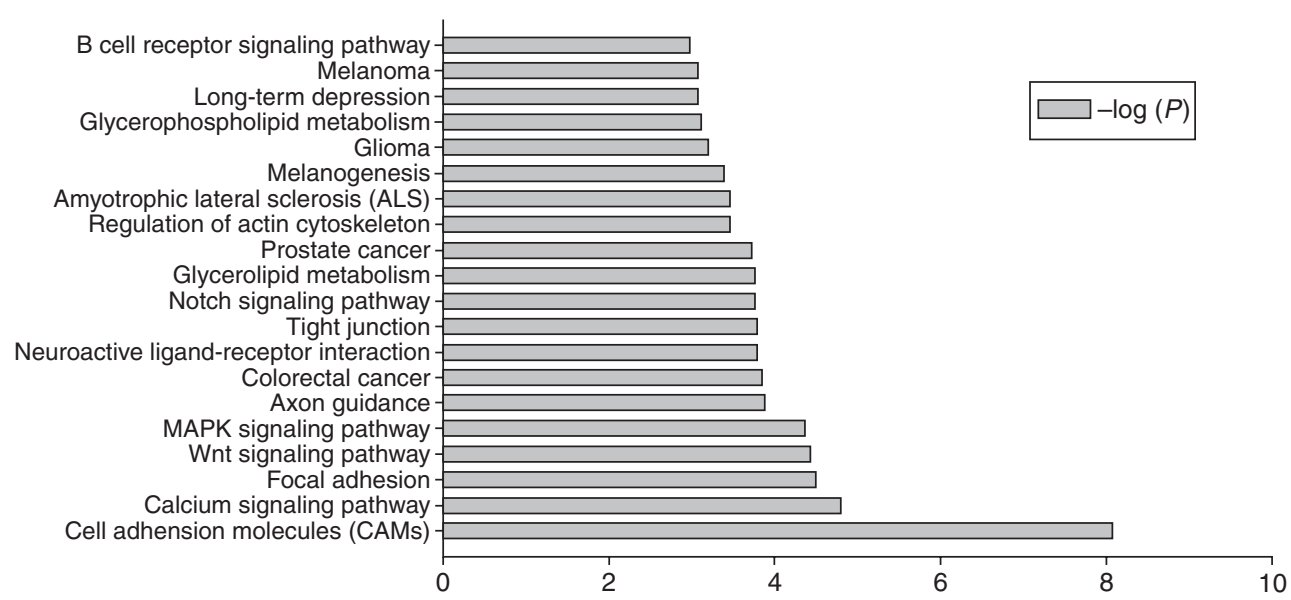

Figure 6 Result of GO and pathway analysis of target genes predicted by hsa-mir-184. (A) Main biological processes of genes targeted by hsa-mir-184. (B) Main pathway involved by genes targeted by hsa-mir-184.

for the target genes of has-miR-184 (Fig. 6). The most relevant KEGG pathway was cell adhesion molecules (Fig. 6B), and GO analysis also indicated that hsa-miR-184 regulated cell adhesion (Fig. 6A). Coincidentally, GO and pathway analysis of other differentially expressed miRNAs demonstrated that they also regulated cell adhesion via target genes (Figs 4 and 5). For example, the most significant KEGG pathway regulated by miRNAs which was down expressed in the villus of RSA patients was focal adhesion (Fig. 5B). High expression of miRNAs in the decidua of RSA patients was also involved in the regulation of cell adhesion and focal adhesion (Figs 4C and 5C). We also found the regulated relationship between hsa-miR-187 and focal adhesion (Fig. 5A). From above results we know, all the differentially expressed miRNAs are involved in the regulation of adhesion function. Therefore, we speculate that miRNA could affect cell adhesion and then regulate the occurrence of RSA.

Although the relative importance of immunological factors in human reproduction remains controversial, substantial evidence suggests that the leukaemia inhibitory factor (LIF), progesterone-induced blocking factor (PIBF), HLA-DPB1 and CD69 contribute to RSA. From targets' prediction data, we found upregulated miRNAs (hsa-miR-519a-1 and hsa-miR-520h) in decidua and low expressed miRNA (hsa-miR-520f) in villus of RSA patients, all target to LIF (Supplementary Table 3, see section on supplementary data given at the end of this article), substantial evidence suggests that LIF contributes to reproductive failure (Choudhury \& Knapp 2001). In decidua, PIBF is known as a target gene of hsa-miR 520h (Supplementary Table 3), and PIBF can mediate progesterone-induced suppression of decidual lymphocyte cytotoxicity (Laskarin et al. 2002). We predicted HLA-DPB1 as a target gene of hsa-miR-520f, which was low expressed in villus (Supplementary Table 3), and just as Takakuwa et al. (2003) reported HLA-DPB1 might be a risk allele for unexplained recurrent abortion in the Japanese population. From target gene results (Supplementary Table 3), hsa-miR-519a-1 upregulated in the decidua of RSA patients could target $3^{\prime}$ UTR of CD69; therefore, intracellular expression of CD69 in endometrial and 
peripheral T cells represents a useful marker in women with recurrent miscarriage (Ramhorst et al. 2003).

Previous study has demonstrated that the angiogenesis in NP is very critical (Reynolds et al. 1992, Chatzaki et al. 2001). Kwang-Hyun Baek compared the gene expression level of angiogenesis-related genes in chorionic villi of RSA patients with those of normal controls (Choi et al. 2003). The result indicated that the lower expression of angiogenesis-related genes is associated with RSA (Choi et al. 2003). In our study, high expression of hsa-miR-519a and hsa 520h in decidua can downregulate matrix metalloproteinase 2 (MMP2; Supplementary Table 3). It has been demonstrated that fibronectin upregulates MMP2 in human T-lymphocytemediated adhesion and migration (Esparza et al. 1999), and there is increased frequency of $\mathrm{T}$ cell adhesion to extracellular matrix fibronectin in women suffering from RSA (Jerzak et al. 2000). MMPs were also involved in promoting the invasiveneness of trophoblasts and decidual stromal cells. Abnormal low expression of MMPs at the maternal-fetal interface will lead to the deficiency of trophoblasts invasion, and further contribute to the origin of RSA (Du et al. 2012, Nissi et al. 2013). In addition, the other angiogenesis-related genes, such as integrin-alphaV, transforming growth factor $\beta 2$ (TGF $\beta 2$ ), TGF $\beta$ receptor 1 (TGF $\beta R 1$ ), vascular endothelial growth factor $A$ and TGF $\beta$ R3 downregulated by highly expressed has-miR-520h, hsa-miR-519a and hsa-miR-522 in decidua (Supplementary Table 3). Based on these results, aberrant angiogenesis may be partially attributed to overexpressed miRNAs in the decidua of RSA patients.

From the above results, we can speculate a network of miRNA regulating RSA. Firstly, all significantly differentially expressed miRNAs affect pregnancy by regulating the adhesion function. In addition, part of the miRNAs can lead to the occurrence of RSA via regulating the expression of immunological factors and angiogenesis-related genes. All of these miRNAs act together to regulate a variety of physiological functions, which leads to RSA. However, all of these predictions are merely a starting point for the study of miRNAs in RSA, and the exact functions of these miRNAs identified in this article are still waiting for the experimental evidence to verify.

\section{Supplementary data}

This is linked to the online version of the paper at http://dx.doi. org/10.1530/REP-14-0095.

\section{Declaration of interest}

The authors declare that there is no conflict of interest that could be perceived as prejudicing the impartiality of the research reported.

\section{Funding}

This work is supported by grants from the Priority Academic Development Program of Jiangsu Higher Education Institutions (PAPD), Program for Changjiang Scholars and Innovative Research Team in University (PCSIRT and IRT1075), Jiangsu Key Laboratory of Infection and Immunity, Institutes of Biology and Medical Sciences of Soochow University, and intramural research funding to J Zhang (Q413401810 and Q313406711) from Soochow University, National Natural Science Foundation of China to J Zhang (31270939), F Xia (31200660) and F Dong (81300553), Natural Science Foundation of Jiangsu Province to J Zhang (BK2012617), Key University Science Research Project of Jiangsu Province to J Zhang (13KJA310004). National Natural Science Foundation of China (31170870) to L Jin; Training Program for excellent academic leaders of Shanghai Health System XBR2013093 to L Jin.

\section{References}

Bulla R, de Guarrini F, Pausa M, Fischetti F, Meroni PL, De Seta F, Guaschino S \& Tedesco F 1999 Inhibition of trophoblast adhesion to endothelial cells by the sera of women with recurrent spontaneous abortions. American Journal of Reproductive Immunology 42 116-123.

Chaiworapongsa T, Romero R, Yoshimatsu J, Espinoza J, Kim YM, Park K, Kalache K, Edwin S, Bujold E \& Gomez R 2002 Soluble adhesion molecule profile in normal pregnancy and pre-eclampsia. Journal of Maternal-Fetal \& Neonatal Medicine 12 19-27. (doi:10.1080/jmf.12. 1.19.27)

Chatzaki E, Makrigiannakis A, Margioris AN, Kouimtzoglou E \& Gravanis A 2001 The Fas/FasL apoptotic pathway is involved in $\kappa$-opioid-induced apoptosis of human endometrial stromal cells. Molecular Human Reproduction 7 867-874. (doi:10.1093/molehr/7.9.867)

Choi HK, Choi BC, Lee SH, Kim JW, Cha KY \& Baek KH 2003 Expression of angiogenesis- and apoptosis-related genes in chorionic villi derived from recurrent pregnancy loss patients. Molecular Reproduction and Development 66 24-31. (doi:10.1002/mrd.10331)

Choudhury SR \& Knapp LA 2001 Human reproductive failure I: immunological factors. Human Reproduction Update 7 113-134. (doi:10.1093/humupd/7.2.113)

Du MR, Zhou WH, Piao HL, Li MQ, Tang CL \& Li DJ 2012 Cyclosporin A promotes crosstalk between human cytotrophoblast and decidual stromal cell through up-regulating CXCL12/CXCR4 interaction. Human Reproduction 27 1955-1965. (doi:10.1093/humrep/des111)

Esparza J, Vilardell C, Calvo J, Juan M, Vives J, Urbano-Marquez A, Yague J \& Cid MC 1999 Fibronectin upregulates gelatinase B (MMP-9) and induces coordinated expression of gelatinase $\mathrm{A}$ (MMP-2) and its activator MT1-MMP (MMP-14) by human T lymphocyte cell lines. A process repressed through RAS/MAP kinase signaling pathways. Blood $\mathbf{9 4}$ 2754-2766.

de Hoon MJ, Imoto S, Nolan J \& Miyano S 2004 Open source clustering software. Bioinformatics 20 1453-1454. (doi:10.1093/bioinformatics/ bth078)

Jeon YJ, Choi YS, Rah H, Kim SY, Choi DH, Cha SH, Shin JE, Shim SH, Lee WS \& Kim NK 2012a Association study of microRNA polymorphisms with risk of idiopathic recurrent spontaneous abortion in Korean women. Gene 494 168-173. (doi:10.1016/j.gene.2011.12.026)

Jeon YJ, Kim SY, Rah H, Choi DH, Cha SH, Yoon TK, Lee WS, Shim SH \& Kim NK 2012b Association of the miR-146aC $>$ G, miR-149T $>$ C, miR-196a2T $>C$, and miR-499A $>$ G polymorphisms with risk of spontaneously aborted fetuses. American Journal of Reproductive Immunology 68 408-417. (doi:10.1111/aji.12005)

Jerzak M, Rechberger T \& Gorski A 2000 Intravenous immunoglobulin therapy influences $\mathrm{T}$ cell adhesion to extracellular matrix in women with a history of recurrent spontaneous abortions. American Journal of Reproductive Immunology 44 336-341. (doi:10.1111/j.8755-8920. 2000.440603.x) 
Konijnenberg A, Stokkers EW, van der Post JA, Schaap MC, Boer K, Bleker OP \& Sturk A 1997 Extensive platelet activation in preeclampsia compared with normal pregnancy: enhanced expression of cell adhesion molecules. American Journal of Obstetrics and Gynecology 176 461-469. (doi:10.1016/S0002-9378(97)70516-7)

Laskarin G, Tokmadzic VS, Strbo N, Bogovic T, Szekeres-Bartho J, Randic L, Podack ER \& Rukavina D 2002 Progesterone induced blocking factor (PIBF) mediates progesterone induced suppression of decidual lymphocyte cytotoxicity. American Journal of Reproductive Immunology 48 201-209. (doi:10.1034/j.1600-0897.2002.01133.x)

Lee RC, Feinbaum RL \& Ambros V 1993 The C. elegans heterochronic gene lin-4 encodes small RNAs with antisense complementarity to lin-14. Cell 75 843-854. (doi:10.1016/0092-8674(93)90529-Y)

Livak KJ \& Schmittgen TD 2001 Analysis of relative gene expression data using real-time quantitative PCR and the $2(-$ Delta Delta $\mathrm{C}(\mathrm{T}))$ method. Methods 25 402-408. (doi:10.1006/meth.2001.1262)

Lyall F, Hayman RG, Ashworth JR, Duffie E \& Baker PN 1999 Relationship of cell adhesion molecule expression to endothelium-dependent relaxation in normal pregnancy and pregnancies complicated with preeclampsia or fetal growth restriction. Journal of the Society for Gynecologic Investigation 6 196-201. (doi:10.1016/S10715576(99)00014-3)

Malgorzata J \& Gorski A 1998 T cell adhesion to the extracellular matrix proteins as a determinant of pregnancy success or failure. Immunology Letters 63 135-140. (doi:10.1016/S0165-2478(98)00067-4)

McCallie B, Schoolcraft WB \& Katz-Jaffe MG 2010 Aberration of blastocyst microRNA expression is associated with human infertility. Fertility and Sterility 93 2374-2382. (doi:10.1016/j.fertnstert.2009.01. 069)

Nissi R, Talvensaari-Mattila A, Kotila V, Niinimaki M, Jarvela I \& Turpeenniemi-Hujanen T 2013 Circulating matrix metalloproteinase MMP-9 and MMP-2/TIMP-2 complex are associated with spontaneous early pregnancy failure. Reproductive Biology and Endocrinology 112. (doi:10.1186/1477-7827-11-2)

Pan Q, Luo X, Toloubeydokhti T \& Chegini N 2007 The expression profile of micro-RNA in endometrium and endometriosis and the influence of ovarian steroids on their expression. Molecular Human Reproduction 13 797-806. (doi:10.1093/molehr/gam063)
Pandey MK, Rani R \& Agrawal S 2005 An update in recurrent spontaneous abortion. Archives of Gynecology and Obstetrics 272 95-108. (doi:10.1007/s00404-004-0706-y)

Rai R \& Regan L 2006 Recurrent miscarriage. Lancet 368 601-611. (doi:10.1016/S0140-6736(06)69204-0)

Ramhorst R, Garcia V, Agriello E, Corigliano A, Etchepareborda E, Irigoyen M, Pasanante G \& Fainboim L 2003 Intracellular expression of CD69 in endometrial and peripheral T cells represents a useful marker in women with recurrent miscarriage: modulation after allogeneic leukocyte immunotherapy. American Journal of Reproductive Immunology 49 149-158. (doi:10.1034/j.1600-0897.2003.00021.x)

Reynolds LP, Killilea SD \& Redmer DA 1992 Angiogenesis in the female reproductive system. FASEB Journal 6 886-892.

Sun L, Xie H, Mori MA, Alexander R, Yuan B, Hattangadi SM, Liu Q, Kahn CR \& Lodish HF 2011 MiR193b-365 is essential for brown fat differentiation. Nature Cell Biology 13 958-965. (doi:10.1038/ncb2286)

Takakuwa K, Adachi H, Hataya I, Ishii K, Tamura M \& Tanaka K 2003 Molecular genetic studies of HLA-DRB1 alleles in patients with unexplained recurrent abortion in the Japanese population. Human Reproduction 18 728-733. (doi:10.1093/humrep/deg188)

Wang X, Li B, Wang J, Lei J, Liu C, Ma Y \& Zhao H 2012 Evidence that miR-133a causes recurrent spontaneous abortion by reducing HLA-G expression. Reproductive Biomedicine Online 25 415-424. (doi:10. 1016/j.rbmo.2012.06.022)

Wightman B, Ha I \& Ruvkun G 1993 Posttranscriptional regulation of the heterochronic gene lin-14 by lin-4 mediates temporal pattern formation in C. elegans. Cell 75 855-862. (doi:10.1016/0092-8674(93)90530-4)

Zhang Y, Diao Z, Su L, Sun H, Li R, Cui H \& Hu Y 2010 MicroRNA-155 contributes to preeclampsia by down-regulating CYR61. American Journal of Obstetrics and Gynecology 202 e461-e467. (doi:10.1016/ j.ajog.2010.01.057)

Received 19 February 2014

First decision 12 March 2014

Revised manuscript received 20 March 2014

Accepted 31 March 2014 